\title{
Acceptance of telepathology in daily practice
}

\author{
Thomas Mairinger \\ Department of Pathology, University of Innsbruck, \\ Muellerstrasse 44, A-6020 Innsbruck, Austria \\ Tel.: +43512507 3661; Fax: +435125820 88; \\ E-mail:Thomas.Mairinger@uibk.ac.at
}

The availability of pathology services differs greatly in our environment. Although pathology would be especially suitable for being practised at a distance by transporting digital image information, the spread of telepathology into everyday work still is relatively slow.

The article describes the situation of diffusion of this innovative technology by reviewing the literature and discussing this in context to data based on questionnaires dealing with the acceptance of telepathology. The current situation of telepathology can be discussed by five items for innovation spead: (1) communication and influence; (2) economic costs and benefits; (3) knowledge barriers and learning; (4) feasibility of techniques offered for the demands of the users; (5) clarification of the legal status and other factors concerning international collaboration. All these head lines do not represent realistic obstacles for the more widespread use of telepathology. The real drawbacks may therefore be found behind certain professional habits of pathologists. The most important causes may be that (a) telediagnosis is not as easy as it may seem at the first glance; (b) telepathology is seen as a potential highway to a world-wide competition of pathology service providers. As soon as these mostly unjustified prejudices are corrected and telepathology is percepted as additional technique in pathology, it will become a diagnostic tool as common and as useful as the telephone.

Keywords: Telepathology, social influence, cost/benefit ratio, Austria questionnaire

\section{Introduction}

The availability of medical services differs greatly between different countries and continents [10,12]. This is especially true for pathology, where many areas are remarkably underserved (Table 1).

Surgical pathology is mainly based on the interpretation of morphological changes by visual examination of histological slides. In other words, the basis of histopathology is the recognition of pathological changes by means of optical media (eye, micro-
Table 1

Relation between population size and numbers of inhabitants in selected countries

\begin{tabular}{lcc}
\hline Country & Inhabitants (mio) & Pathologists \\
\hline Laos & 5 & 10 \\
Thailand & 60 & 250 \\
Germany & 80 & 1500 \\
Austria & 7 & 250 \\
\hline
\end{tabular}

Source: DIAGAID - Project application, G. Stauch, personal communication.

Table 2

Summary of the number of hits during the literature research

\begin{tabular}{lcc}
\hline & Telemedicine & Telepathology \\
\hline Legal & 153 & 9 \\
Economics & 861 & 18 \\
Acceptance & 94 & 9 \\
\hline
\end{tabular}

scope). If this statement hold true, pathology would be extraordinarily suitable for being practised at a distance just by transporting (digital) image information, i.e., telepathology would be applied.

However, the reality of the telepathology used in practice, differs broadly from the situation to be expected [15]. Although the technological basics of telepathology are widely known and the application has been demonstrated to work surroundings wide variety of technical reliably and sufficiently fast in a real telepathology applications in routine work are still rare $[3,9,14,19,20,24]$.

There is still a paucity of research on the diffusion of telemedicine, which is a relatively new and complex phenomenon $[10,12,22]$. As a new medical practice, telemedicine can be conceptualized as an innovative process [22]. To analyze the innovation and diffusion process in the literature may, therefore, be used to study why the spread of telemedicine still is (relatively) low. In combination with data about the attitude of various potential end user groups one may get from these results an idea about the main obstacles that hinder a widespread use of telepathology in daily practice.

This article tries to give a critical overview of the acceptance of current telepathology technologies within the potential end users and possible strategies to im- 
prove the acceptance and subsequently the implementation speed of this technology.

\section{Materials and methods}

Data obtained by three questionnaires and an extensive literature research were analyzed.

The basic search tool for the review of the literature was the PubMed System by the National Institute of Health (http://www.ncbi.nlm.nih.gov/PubMed). The key words used for research covered telepathology as well as telemedicine in general. Special emphasis was given to papers dealing with "acceptance" and the "legal" situation as well as "economical" topics.

\subsection{Questionnaires used}

The results of three questionnaires were used for this overview: Full results of these questionnaires are published elsewhere $[7,15,16]$.

These questionnaires were sent a total of 300 physicians in Spain, Central Europe and Ireland, the members of the Austrian Society of Pathology and 300 students attending a students conference in Tenerife/Spain. All data were acquired between 1995 and 1997.

\section{Results}

In the recent literature, 283 articles dealing with Telepathology can be found.

The search for the keywords "telemedicine and legal" revealed 153 papers, whereas "telepathology and legal" results in just 9 articles.

861 papers deal with "telemedicine and economics", out of those 18 can be found by the keywords "telepathology" and "economics".

"Telemedicine and acceptance" is discussed in 94 papers, whereas only 9 articles deal with "telepathology" and "acceptance" respectively "attitude".

Within the subject telepathology, most papers analyze first opinion telediagnostics (mainly frozen section services at a distance), far less papers expert advice (second opinion). Only 5 articles focus on "teleeducation" or "telelearning" in medicine.

From the questionnaires listed above, answers to some selected questions are listed in Tables 3 and 4. The complete results can be read in [16].
Table 3

Potential benefits of telemedicine as seen by Austrian pathologists (full results published elsewhere)

\begin{tabular}{lcc}
\hline & Enormous benefit & No benefit \\
\hline First opinion diagnostics & $22 \%$ & $29 \%$ \\
Second opinion diagnostics & $73 \%$ & $3 \%$ \\
Clinicopathological conferences & $59 \%$ & $7 \%$ \\
Education/telelearning & $69 \%$ & $5 \%$ \\
\hline
\end{tabular}

Table 4

Potential difficulties of telemedicine as seen by Austrian pathologists (full results published elsewhere)

\begin{tabular}{lccc}
\hline & $\begin{array}{c}\text { Very problematic } \\
\text { or problematic }\end{array}$ & $\begin{array}{c}\text { Minor } \\
\text { problems }\end{array}$ & $\begin{array}{c}\text { No } \\
\text { problem }\end{array}$ \\
\hline Costs/reimbursement & $49 \%$ & $41 \%$ & $6 \%$ \\
$\begin{array}{c}\text { Time for the consulted } \\
\text { expert }\end{array}$ & $35 \%$ & $56 \%$ & $3 \%$ \\
Responsibility/liability & $67 \%$ & $16 \%$ & $4 \%$ \\
\hline
\end{tabular}

\section{Discussion}

The results of three questionnaires and an extensive literature search were used to analyze the basic obstacles for a more rapid spread of telemedicine technology in routine pathology. One may define three metaphors that dominate the innovation diffusion [22]:

(1) communication and influence,

(2) economic costs and benefits,

(3) knowledge barriers and learning.

In the case of telepathology, these metaphores may be extended by

(4) feasibility of techniques offered for the demands of the users,

(5) clarification of the legal status and other factors concerning international collaboration.

\subsection{Communication and influence}

The level of knowledge about telemedicine is quite high in the investigated groups of potential end users. The number of articles published dealing with telepathology topics is numerous, and relevant data can be found in specialized general pathology journals. Big companies like Leica, Olympus, Nikon, Zeiss and others display their telepathology systems at pathology conferences. Thus, the level of knowledge and information concerning telepathology is high in the field of pathology [15]. The potential role of telepathology is generally judged of high importance $[15,16]$. 
When going more into detail, this positive overall attitude looses some of its glamour: Enormous benefit on first opinion diagnostics is expected by less than a quarter of pathologists. Nearly $75 \%$, in addition, expect great benefits for second opinion diagnosis (Table 3). This is in remarkable contrast to the picture drawn by the literature published, where the ratio of papers dealing with first opinion versus second opinion diagnosis is clearly in favor of first opinion diagnosis, especially focussing on the feasibility of frozen section diagnosis at a distance. As the technical requirements for rendering a primary diagnosis at a distance are high, the companies expect a potentially great-market and focus, therefore, primarily on this application. On the other hand, rendering a primary diagnosis raises problems of responsibility of sampling and specimen preparation at a distance. It is felt, that the control of pathologist rendering the diagnosis is lost. Macroscopical examination of specimens by other medical specialists is for many pathologists an unacceptable situation. They have the impression that the person responsible for the diagnosis looses the control over process. This seems to be a minor problem in second opinion diagnosis [15].

\subsection{Economic costs and benefits}

\subsubsection{Costs of telepathology}

Ready to use systems. One would assume that exact data are known on the economic costs of telepathology. However, this is only true for purchasing of a dedicated piece of equipment like a robotic microscope. Much less detailed knowledge is available for the real overall costs of telepathology (only a few articles have been published which deal with this topic (Table 2)). Rarely the time of all persons involved at both sides of the communication line (physicians, technicians and laboratory personnel as well as people maintaining the system) is analyzed [4,11]. An exact estimation of the costs to be expected in a routine setting has to be calculated for each system specifically.

Custom made, do it yourself systems. Another approach to telemedicine is the pioneer's approach by "do it yourself" - strategies. Exact cost calculations are not possible, as these systems are based on equipment that originally has been purchased for different tasks and is "by chance" suitable for telepathology. This could include various hardware components such as color CCD-cameras, etc. The same holds true for the costs of communication: An Internet-connection is often already present and not exclusively installed for telepathology. Such systems are most often used for still image "store and forward" telepathology approaches. They can be regarded as cheap but valuable solutions for entering the field [11-14].

\subsubsection{Telepathology benefits}

Similar to the difficult cost calculation in telepathology service it is also difficult to assess the benefits of a telepathology service. The calculation of the benefit is extremely difficult. This benefit is not only achieved by the saving of transportation costs of the specimen, e.g., intraoperative taxi transport of tissue or parcel services for sending slides to experts for a second opinion. Potential more benefit is related to a telepathology service: peripheral hospitals that need frozen section services will send their specimens to an institute that can provide these services. A frozen section service may well be an advantage in competition between different pathology departments. Similar, clinico-pathological conferences can be held by videoconferencing, saving time for all participants and avoiding long travels. The same holds true for the more rapid availability of a second opinion, which can be obtained within 12 days when using telepathology services. Physicians will probably prefer to collaborate with pathologists who can provide their services in a minimum period of time. The image of pathologists using telepathology is expected to improve by use of this technology $[15,16]$.

Bejond all these factors that may lead to indirect benefits for the "telepathologist" there is the still unsolved matter of direct reimbursement of telepathology services (Table 4). As long as these time-consuming and technologically demanding services are not reimbursed, telepathology will stay in its status of a interesting and personally valuable tool for a small group of interested pathologists.

The introduction of a reimbursement system that allows national as well as international reimbursement would immediately increase the acceptance of this technology. It is a paradox on that just this potential reimbursement of telepathology services turns out to be one of the most resistant obstacles for a more general acceptance in Europe.

As soon as an international reimbursement system will be established, all pathologists were part of a world wide "Pathology Service Market", where expertise could be bought worldwide at the best ratio service versus price. This outlooks may well be an obstacle against more directed attempts for establishing national as well as international reimbursement modalities. 


\subsection{Knowledge barriers and learning}

Knowledge barriers have been a remarkable problem in the pioneer days of telematics in medicine. It is a well known fact that the users have to get used to new technologies to be able to perform them well and feel good when using them $[6,11,22]$. Pathologists know about the basics of telepathology, however, often not have tried to make a remote diagnosis by them selves or select images for making a diagnosis at a distance. Nevertheless, the dramatic increase of the use of personal computers and working on a screen facilitate the use of telemedicine devices. Almost all physicians are capable of operating a computer, and technology barriers will disappear soon [3]. As soon as telepathology is understood as a special technique in the diagnostic procedure, pathologists will improve their capabilities by attending courses, workshops, etc., thus lowering the knowledge barriers rapidly [2,13,23].

\subsection{Feasibility of techniques offered for the demands of the users}

Without doubt this point is one of the most critical aspects for the implementation of this technology. It may be well understood that pathologists in former years did not want to make diagnoses on small, low resolution images, as they are used to the images seen through their microscope. When looking at the results of the questionnaire among Austrian pathologists it is remarkable that almost all participating colleagues favor high technology solutions including a robotic microscopy and live images [15]. To my opinion, this may be related to the fact that telemedicine is still nearly exclusively associated with applications favoring real time telepathology such as frozen section services. Nevertheless, the majority of pathologists is aware of the fact that the technological development has already solved or is at least about to solve the problems of image quality and available bandwidth [8,11]. Technical problems will not remain a limiting factor for the spread of telepathology.

\subsection{Clarification of the legal status of a telepathology consultation}

One of the problems judged as being one of the most important drawbacks for telepathology has been the legal status of teleconsultation (Table 4). This is difficult to understand, as the fears seem to be unjustified.
Many articles have been published, describing in detail the responsibilities in telepathology consultation. This has been investigated for many countries and legal systems $[1,17,18,21]$. International cooperations, however, have not been extensively studied until now.

\section{Conclusion}

From the presented data it remains unclear why telepathology has not spread with more intensity until now. When using classical approaches for the assessment of innovation diffusion, all demands from the above mentioned metaphors are fulfilled, and no obvious rationale can be identified as potential obstacle for everyday's use of telepathology.

The real reasons may be found behind the curtain of tradition and professional habits of pathologists. Strategies to improve the acceptance of telepathology should respond to these factors:

(1) Telepathology is usually identified with remote frozen section services. The process of remotely rendering a primary diagnosis is probably the most spectacular example of telepathology. However, it is just a small segment when performing this technology. This segment was used to raise attraction to this field, but from today viewpoint it turned out to be not very productive. As a consequence most pathologists consider telepathology equal to remote frozen section services, affected by high costs and legal uncertainties. The far more beneficial use of telematic technology such as second opinion $[2,5]$ or telelearning $[8,12]$ should be more in focus. The exchange of knowledge between experts when discussing difficult cases by means of telepathology is not affected by problems of sampling, etc., and can be realized without major expenses. Remote frozen section service should be regarded as a subtopic of telepathology.

(2) Telediagnosis is a process performed not as easy as it may thought. Rendering diagnoses on screen seems to be trivial at a first glance. To render a diagnosis on screen, however is a process that is different from the way pathologists are used to work. Even though the image quality, etc. is found to be sufficient for diagnosis, and the purely rational approach tells us that the human eye cannot percept higher image qualities as those which are already present. Pathologists do not feel well when using the monitor instead of the microscope. It has to become accepted that rendering diagnoses on a screen is a process that has to be learned 
and practiced similar to other techniques [6]. Only if this fact is generally accepted, the use of telepathology will not start with the frustating experience of feeling insecure when rendering diagnoses on a screen.

(3) Telepathology is time-consuming and not reimbursed. Besides all academic interest and fondness of new technologies one has to be aware of the fact that the use of this technology is more time-consuming than traditional microscopy. This is due to the fact that the exchange of image information is still a tedious task when using wide area networks like the Internet. The time to come to a diagnostic decision is longer than using a conventional microscopy. As most pathologists are urged to earn money by their daily routine diagnostic work, the relatively long time needed for telediagnosis makes the use of telepathology less attractive. During the start of integrating this procedure is difficult to implement in daily routine work, when factors of insecurity about the technique may need further time resources.

The low attractivity of using telepathology due to the relatively high amount of time needed is severely aggravated by the fact that in most countries telepathology is a service that is not reimbursed. This is not attractive for the introduction of telepathology, especially when thinking of using expensive live remotely controlled systems. However, as soon as pathologists start to understand telepathology not only as a remote primary diagnosis where expensive equipment is necessary, but rather as a tool to exchange images instead of blocks or sections for second opinion or teaching/learning purposes, the question of reimbursement becomes less important.

(4) Telepathology is the potential highway to a worldwide competition of pathology service providers. One of the fears that are often not expicitely stated when the problems of accepting telepathology are discussed is closely related to the high expectations on this method by pathologists. Many colleagues have the feeling that the spread of telematics in pathology would open up the highway of globalisation of pathology service, i.e., the fact that any clinician could choose his/her pathologist out of all pathology experts around the world. In this use, some colleagues fear that they would loose physicians submitting their specimens and subsequently loosing their place in the medical concert. As it is obvious that the technology is capable of being used for such purposes, many colleagues try to withdraw telepathology instead of implementing it in their routine work. To my opinion, this is the wrong way to deal with telematics in pathology: The reasons for a physician to trust "his" pathologist are far beyond the simple fact of being a well known or famous diagnostic capacity. Factors like good personal contact, careful diagnosis and reliable connections between the physician and the pathologist are by far more important and superior reasons to choose the pathologist on site. Telepathology should be percepted as a possibility to improve everybody service to the clinicians in order to speed up and facilitate the spread of expertise, in getting a second opinion and improved quality of diagnoses.

In summary, no real barriers seem to exist that would explain the slow spread of telematics in pathology. As soon as telepathology is percepted as additional technique in pathology to rather be used for rapid acquisition of a second opinion or improved facilities for selfeducation [8] endless as a threat of loosing the economic basis by entering in a global market, telepathology should become a tool as common as the telephone to be used by the pathologists for the benefit of the patient.

\section{References}

[1] F.A. Allaert and L. Duserre, Legal requirements for teleassistance and tele-medicine, Medical Informatics 8 (1995), 1593-1595.

[2] C.A. Beltrami and V. Della Mea, Second opinion consultation through the Internet. A three years experience, Adv. Clin. Pathol. 2(2) (1998), 146-148.

[3] V. Della Mea and C.A. Beltrami, Diagnostic telepathology through the Internet, Histopathology 33(5) (1998), 485.

[4] V. Della Mea, D. Cortolezzis and C.A. Beltrami, The economics of telepathology - a case study, J. Telemed. Telecare 6 (2000), 168-169.

[5] V. Eusebi, I. Foschini, S. Erde and J. Rosai, Transcontinental results in surgical pathology via the Internet, Human Pathol. 28 (1997), 22-29.

[6] O. Ferrer-Roca, S. Pitti, A.D. Cardama, S. Makidou, C. Maseo, A. Ramos and H. Coen, Factors influencing distant teleevaluation in cytology, pathology, conventional radiology and mammography, Analyt. Cell. Pathol. 10 (1996), 13-23.

[7] A. Gschwendtner, T.T. Netzer, B. Mairinger and T. Mairinger, What do students think about telemedicine?, J. Telemed. Telecare 3 (1997), 1-3.

[8] D.G. Jameson, P. O'Hanlon, S. Buckton and M. Hobsley, Broadband telemedicine: teaching on the information superhighway, J. Telemed. Telecare 1 (1995), 111-116.

[9] K. Kayser, M. Drlicek and W. Rahn, Aids of telepathology in intraoperative histomorphological tumor diagnosis and classification, In vivo 7 (1993), 379-382. 
[10] K. Kayser and C. Kayser, Telepathology - aspects of social influence and quality control, Elec. J. Pathol. Histol. 3(3) (1996), No. 963-04.

[11] K. Kayser, G. Kayser and S. Zink, New technical aspects in telepathology, Elec. J. Pathol. Histol. 6(3) (2000), No. 003-04.

[12] K. Kayser, J. Szymas and R. Weinstein, Telepathology Telecommunication, Electronic Education and Publication in Pathology, Springer, Heidelberg, New York, 1999.

[13] K. Kayser, Telemedizin, Wiener Klin. Wschr. 108 (1996), 932 940.

[14] K. Kayser, Telepathology in Europe. Its practical use, Arch Anat. Cytol. Pathol. 43 (1995), 196-199.

[15] T. Mairinger, T. Netzer, W. Schoner and A. Gschwendtner, Pathologist's attitude to implementing telepathology, J. Telemed. Telecare 4 (1998), 41-46.

[16] T. Mairinger, O. Ferrer-Roca, C. Gabl, P. Dervan and G. Mikuz, What do colleagues think of telemedicine? Evaluation of a Telemedicine questionnaire in different European regions, J. Telemed. Telecare 2 (1996), 50-56.

[17] T. Mairinger, T. Netzer, A. Gschwendtner and Ch. Mark, The legal situation of telemedicine in Austria, J. Telemed. Telecare 3 (1997), 154-157.
[18] T. Netzer, T. Mairinger, A. Gschwendtner, G. Mikuz and Ch. Markl, Die rechtliche Lage der Telemedizin in Österreich, Wiener Klin. Wschr. 17 (1996), 555-559.

[19] I. Nordrum, B. Engum, E. Rinde, A. Finseth, H. Ericsson, M. Kearney, H. Stralsberg and T.J. Eide, Remote frozen section service: a telepathology project in northern Norway, Human Pathol. 22(6) (1991), 514-518.

[20] M. Oberholzer, H.R. Fischer, H. Christen, S. Gerber, M. Brühlmann, M. Mihatsch, T. Gahm, M. Famos, C. Winkler and P. Fehr, Telepathology: frozen section image analysis at a distance, Virchows Archiv 426(1) (1995), 3-9.

[21] M. Schiffer, Legal aspect of telepathology, Zetralbl. Pathol. 138 (1992), 393-394.

[22] H. Tanriverdi and C.S. Iacono, Diffusion of telemedicine: A knowledge barrier perspective, Telemed. J. 5(3) (1999), 223244.

[23] R.S. Weinstein, Static image telepathology in perspective, $\mathrm{Hu}$ man Pathol. 27 (1996), 99-101.

[24] R.S. Weinstein, A.K. Bhattachryya, A.R. Graham and J.R. Davis, Telepathology: a ten years progress report, Human Pathol. 28 (1996), 1-7. 


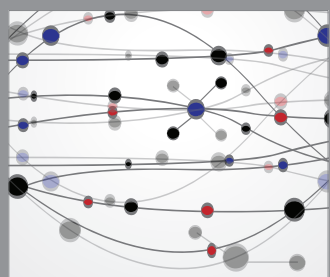

The Scientific World Journal
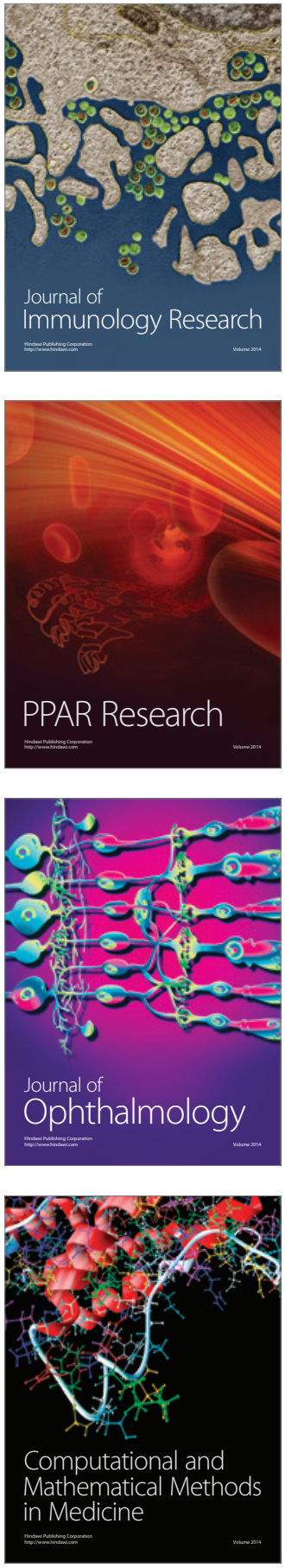

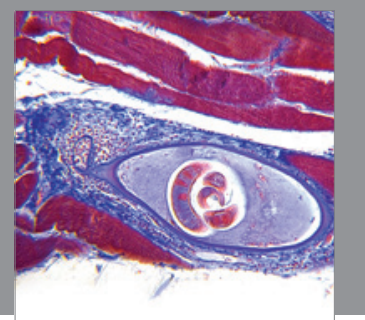

Gastroenterology

Research and Practice


\section{Hindawi}

Submit your manuscripts at

http://www.hindawi.com


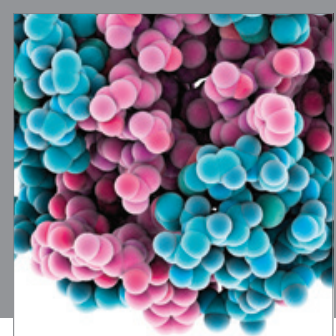

Journal of
Diabetes Research

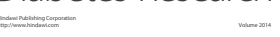

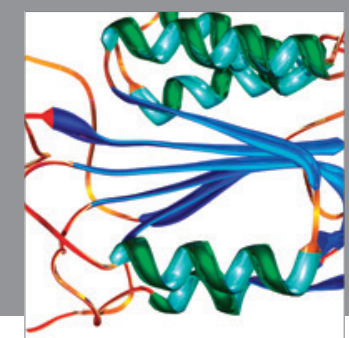

Disease Markers
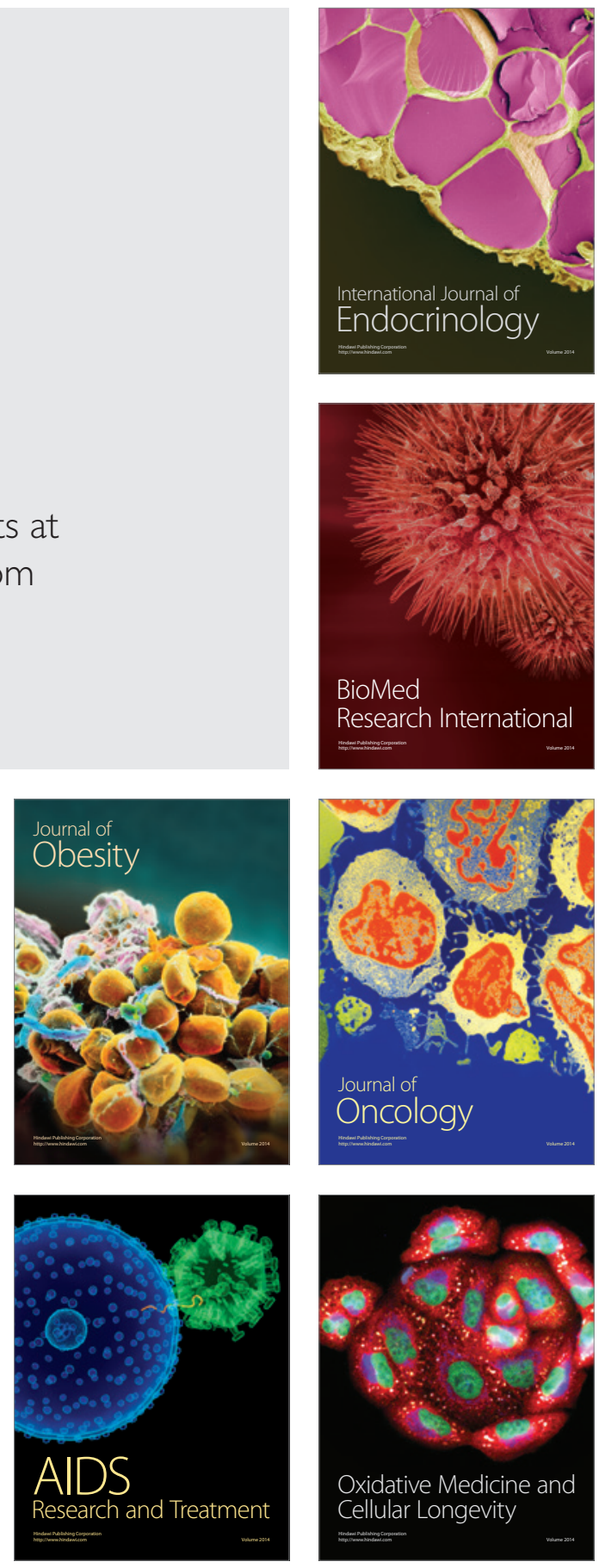